\title{
Tendências de Pesquisas que Utilizam Learning Analytics em MOOCs: um mapeamento sistemático
}

\author{
Napoliana Silva de Souza - PPGIE/UFRGS - souzapoliana2@gmail.com \\ Leandro Krug Wives - PPGIE/UFRGS - wives@inf.ufrgs.br \\ Gabriela Trindade Perry - PPGIE/UFRGS - gabriela.perry@ufrgs.br
}

\begin{abstract}
Resumo. Técnicas de learning analytics têm sido amplamente utilizadas em ambientes MOOCs para realizar diferentes análises educacionais. Nesse contexto, foi conduzido um mapeamento sistemático em quatro bases de dados com o propósito de verificar quais as tendências inerentes ao uso de learning analytics em MOOCs. A busca compreendeu o período de 2012 a 2018, sendo encontradas 1009 pesquisas, e dentre este total 128 estudos foram selecionados. Os resultados revelaram que a análise do engajamento dos estudantes, investigações relativas à conclusão e abandono de cursos, identificação de padrões de comportamentos de interação e aprendizagem, bem como a análise de desempenho dos estudantes são as temáticas mais frequentes.
\end{abstract}

Palavras-chave: MOOCs, learning analytics, tendências.

\section{Research Trends that apply Learning Analytics in MOOCs: a systematic mapping}

\begin{abstract}
Learning analytics techniques have been widely used in MOOCS environments to perform different educational analyzes. In this context, a systematic mapping was conducted in four databases in order to verify the trends regarding the use of learning analytics in MOOCs. The search comprised the period from 2012 to 2018, being found 1009 researches, and of this total 128 studies were selected. The results revealed that the analysis of student engagement, investigations related to the completion and dropout in courses, identification of patterns of interaction and learning behaviors, as well as student performance analysis are the most frequent topics.
\end{abstract}

Keywords: MOOCs, learning analytics, trends.

\section{Introdução}

Massive Open Online Courses (MOOCs) baseiam-se em aprendizagem aberta e em larga escala, geralmente são ofertados por universidades, visando ampliar as possibilidades educacionais e facilitar o acesso à aprendizagem (Joo, So e Kim, 2018).

Os MOOCs têm recebido muitos inscritos em virtude de seu caráter aberto que permite a todas as pessoas interessadas participar de um curso, e esta abertura é orientada a diversos aspectos, tais como a alternativa de o estudante optar ou não pelo recebimento de certificado de participação no curso, bem como a possibilidade de o estudante decidir se permanecerá ou não até o final do curso (Gil-Jaurena e Domínguez, 2018).

Quanto às características, os MOOCs possuem formatos que podem se diferenciar em termos de design instrucional, meios de suportes oferecidos aos estudantes, formas de 
tutoria, e também há diferenças nas tarefas que os alunos precisam realizar (Drachsler e Kalz, 2016), e os conteúdos podem ser de caráter introdutório ou avançado com o propósito de alcançar públicos diversos (Latchem, 2018).

Os MOOCs também possuem uma dimensão social, pois permitem conectar um grande número de alunos que podem interagir e se apoiarem, de modo a fornecer ou buscar suporte no aprendizado, discutir os conteúdos e compartilhar interesses em comum (Cohen e Holstein, 2018). Além disso, as plataformas de MOOCs registram informações referentes à interação dos estudantes por meio de arquivos de logs, gerando novas possibilidades de compreensão do aprendizado a partir de uma perspectiva quantitativa (Lee, 2018).

Em contextos de cursos a distância verifica-se a necessidade de estudar como ocorre o acompanhamento do curso pelo estudante, bem como analisar a participação, interação e realização de tarefas, pois os comportamentos dos estudantes podem indicar necessidades específicas de aprendizagem, evidenciar dificuldades e revelar indícios de abandono do curso (Zielinski e Schmitt, 2015). Nesse contexto, a análise das ações dos estudantes é uma tarefa fundamental para detectar barreiras para a aprendizagem, principalmente em MOOCs que lidam com baixas taxas de conclusão (He et al., 2015).

Ao armazenar vários dados de aprendizagem e de interação, os MOOCs fornecem condições para extrair informações que direcionem a identificação de características comportamentais e indicadores relacionados à aprendizagem (Lu et al., 2017). Desta forma, considerando que as técnicas de Learning Analytics atuam no âmbito de análise de dados de aprendizagem obtidos de registros de interação on-line, esta pesquisa teve 0 objetivo de realizar um mapeamento sistemático da literatura, a fim de levantar estudos com ênfase no uso de Learning Analytics em MOOCs, de modo a identificar as tendências temáticas, e os propósitos gerais de uso de Learning Analytics em cursos dessa natureza.

Este artigo tem a seguinte estrutura: a Seção 2 traz uma perspectiva geral referente às possibilidades das técnicas de learning analytics para os MOOCs. A Seção 3 descreve os procedimentos metodológicos utilizados na condução do mapeamento sistemático. A Seção 4 apresenta os resultados alcançados. Na Seção 5 constam as considerações finais e, posteriormente, são listadas as referências.

\section{Learning Analytics: possibilidades para as plataformas MOOCs}

Os MOOCs são ambientes de aprendizagem on-line que tem se expandido de forma acelerada, e o fato de reunir um grande número de inscritos tem levado a produção de muitos dados, tornando difícil para o professor acompanhar e realizar análises de todos os dados de aprendizagem, sendo necessário buscar auxílios em ferramentas específicas, e as técnicas de Learning Analytics (LA) surgiram como um suporte para as tarefas de análises de aprendizagem on-line (Ruipérez-Valiente et al., 2015).

LA compreende o uso de técnicas ou ferramentas que faz o uso de dados obtidos de estudantes, com o propósito de efetuar análises educacionais, e gerar estratégias que orientem os estudantes em seu percurso de aprendizado e aos instrutores na realização de intervenções pedagógicas (Pursel et al., 2016).

Ambientes virtuais de aprendizagem registram todas as interações dos estudantes, incluindo o número de cliques, páginas visitadas, desempenho em atividades, todos esses 
dados podem ser explorados por meio de LA para fins de análise da aprendizagem, realização de predições referentes ao aprendizado, detecção de sinais de aprendizagem, planejamento de intervenções, permite também integrar métodos diferenciados, tais como: mineração de dados, análise de conteúdo, análise semântica, entre outros (Clow, 2013).

Devido à riqueza dos dados navegacionais e de aprendizagem dos estudantes, as técnicas de LA fornecem práticas de análises que intencionam o alcance de melhorias educacionais, por meio de diversos procedimentos que buscam a implementação de estratégias de recomendação de conteúdos educacionais, personalização dos conteúdos, predição de abandono, realização de testes estatísticos, estudo de variáveis que se correlacionam com o desempenho, entre outras possibilidades (Siemens, 2013).

Técnicas de LA podem ser empregadas em MOOCs para o estudo de diferentes variáveis, tais como a motivação, envolvimento dos estudantes nas atividades on-line, descoberta de elementos ou indicadores comportamentais de conclusão e sucesso em um curso, identificar padrões de interação, descobrir estratégias que contribuam para a permanência dos estudantes no curso (Pursel et al., 2016).

Além dos dados de cliques é possível examinar uma série de outros elementos, incluindo dados demográficos dos estudantes, e as técnicas de LA também podem atuar integradas a outros métodos e áreas do conhecimento, e os resultados alcançados subsidiam novos significados acerca da aprendizagem e suas relações com o comportamento de interação dos estudantes (Crossley et al., 2016).

As diversas possibilidades de uso das técnicas de LA em ambientes MOOCs motivaram a realização desta pesquisa com o intuito de encontrar quais são as tendências de estudos relacionadas a essa temática.

\section{Procedimentos Metodológicos}

Nesta pesquisa foi realizado um mapeamento sistemático da literatura com o intuito de coletar trabalhos que versam sobre o uso de learning analytics em MOOCs. $\mathrm{O}$ mapeamento teve o objetivo de identificar quais são as tendências de pesquisas, e descobrir os propósitos gerais do emprego de LA em MOOCs.

Um mapeamento sistemático é um método que busca obter uma perspectiva ampla sobre uma área de conhecimento, a fim de coletar evidências inerentes a um tópico, bem como dados quantitativos sobre os estudos que existem relacionados a um tema de pesquisa (Kitchenham e Charters, 2007).

Para a condução do mapeamento foram utilizados os itens contidos nos passos de um processo de mapeamento sistemático apresentado por (Petersen et al., 2008): (i) definição das questões de pesquisa; (ii) busca primária com estabelecimento de strings de busca; (iii) definição das bases de dados; (iv) estabelecimento de critérios de inclusão e exclusão; (v) esquema de classificação; (vi) extração de dados.

Na Figura 1 consta o detalhamento da condução do mapeamento, composto por seis passos. Iniciando pela exposição das quatro questões que nortearam a pesquisa. Em seguida foi estabelecida a string de busca, nesse segundo passo optou-se por utilizar um termo genérico: ("learning analytics" OR "LA") AND ("MOOC" OR "massive open online course"), a fim de alcançar um amplo conjunto de estudos. No terceiro passo foram utilizadas 4 bases de dados. Posteriormente, o quarto passo consistiu na definição dos 
critérios de seleção dos estudos, sendo 5 critérios de inclusão e 3 critérios de exclusão. 0 quinto passo voltou-se para a geração de categorias temáticas de pesquisa, sendo empregado 0 procedimento "Keywording" sugerido por (Petersen et al., 2008). Keywording consiste na tarefa de leitura dos resumos dos papers localizados, com o propósito de encontrar palavraschave que contribuam para gerar as categorias temáticas, e caso o resumo não forneça informações suficientes o procedimento alternativo é recorrer à introdução e conclusão (Petersen et al., 2008).

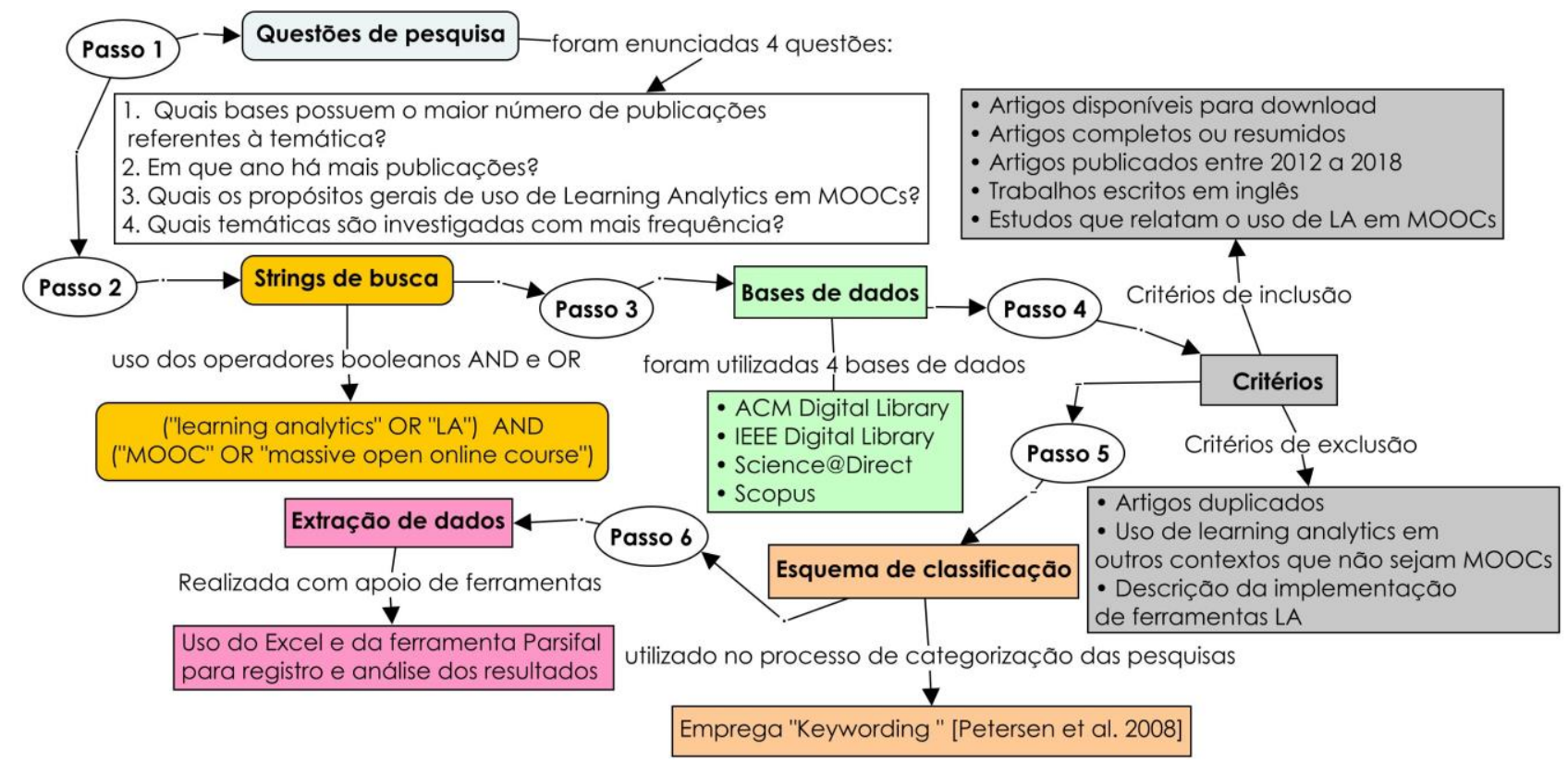

Figura 1. Elementos do mapeamento sistemático.

O mapeamento foi conduzido com o apoio das ferramentas Parsifal ${ }^{1}$ e Excel, que foram fundamentais para os procedimentos de extração dos dados e análise dos resultados, correspondentes ao sexto e último passo metodológico apresentado na Figura 1. Os registros eram formados por 5 atributos: título, autores, local de publicação, ano e classificação temática. Os dados foram analisados em uma dimensão quantitativa, sendo contabilizado o número de publicações por ano, local de publicação e categoria temática. $\mathrm{Na}$ Seção 4 são divulgados os resultados alcançados.

\section{Resultados}

O mapeamento sistemático abrangeu o período de 2012 a 2018, com emprego da string de busca em quatro bases de dados: (i) ACM Digital Library; (ii) IEEE Xplore; (iii) Science@Direct; (iv) Scopus.

Foram selecionados somente artigos completos ou resumidos, escritos em inglês, e que relataram o uso de LA em MOOCs para alguma finalidade. Artigos que somente descrevem a implementação ou apresentação de arquiteturas de ferramentas de learning analytics, bem como as pesquisas que apresentam o uso de learning analytics em contextos que não sejam especificamente MOOCs foram desconsiderados na pesquisa.

\footnotetext{
${ }^{1}$ Parsifal - Disponível em: https://parsif.al/
} 
A Tabela 1 exibe a quantidade de trabalhos retornados e selecionados. Obteve-se o total de 1.009 pesquisas, sendo 119 resultantes da base de dados ACM, 73 estudos encontrados pela IEEE Xplore, 483 localizados pela Science@Direct e 334 estudos são resultados da Scopus.

Tabela 1. Totais de trabalhos encontrados.

\begin{tabular}{l|c|c}
\hline Base de dados & Trabalhos retornados & Selecionados \\
\hline ACM Digital Library & 119 & 40 \\
\hline IEEE Xplore & 73 & 18 \\
\hline Science@Direct & 483 & 19 \\
\hline Scopus & 334 & 51 \\
\hline Totais & 1009 & 128 \\
\hline
\end{tabular}

Conforme encontra-se na Tabela 1, dentre o total de 1.009 pesquisas localizadas, 128 estudos atenderam aos critérios de inclusão, de modo a constituir os objetos de análise para fins de obtenção de respostas às questões formuladas.

A primeira questão de pesquisa voltou-se para o total de publicações: Quais bases de dados possuem o maior número de publicações referentes à temática?. A resposta a essa questão levou em consideração o número de estudos selecionados. A base de dados Scopus apresentou o maior número de publicações segundo os critérios de inclusão, sendo 51 pesquisas selecionadas, em seguida, tem-se a ACM Digital Library com 40 estudos selecionados. A Science@Direct apresentou um número maior de trabalhos retornados, todavia, a maioria não teve aderência ao escopo temático abordado nesta pesquisa.

Os 128 trabalhos selecionados e recuperados pelas bases de dados utilizadas são provenientes de conferências e journals/revistas, tratam-se de publicações de 57 locais diferentes, sendo 31 conferências e 26 journals. Dentre este total foram ranqueados os 15 locais que retornaram a partir de duas publicações sobre a temática, conforme consta na Tabela 2.

Tabela 2. Locais com maior número de publicações.

\begin{tabular}{|l|c|}
\hline Locais & Quantidade de papers \\
\hline $\begin{array}{l}\text { International Conference on Learning } \\
\text { Analytics And Knowledge (LAK) }\end{array}$ & 32 \\
\hline Computers in Human Behavior & 11 \\
\hline Computers \& Education & 7 \\
\hline ACM Conference on Learning @ Scale & 5 \\
\hline Journal of Computer Assisted Learning & 5 \\
\hline Lecture Notes in Computer Science & 3 \\
\hline $\begin{array}{l}\text { Conference on E-Learning \& E-Learning } \\
\text { Technologies }\end{array}$ & 3 \\
\hline $\begin{array}{l}\text { International Conference on Technological } \\
\text { Ecosystems for Enhancing Multiculturality }\end{array}$ & 2 \\
\hline $\begin{array}{l}\text { Conference on User Modeling, Adaptation } \\
\text { and Personalizatio }\end{array}$ & \\
\hline
\end{tabular}




\begin{tabular}{|l|c|}
\hline $\begin{array}{l}\text { IEEE International Conference on Big Data } \\
\text { (Big Data) }\end{array}$ & 2 \\
\hline $\begin{array}{l}\text { Conference on Human Factors in } \\
\text { Computing Systems }\end{array}$ & 2 \\
\hline $\begin{array}{l}\text { Hawaii International Conference on System } \\
\text { Sciences }\end{array}$ & 2 \\
\hline $\begin{array}{l}\text { IEEE Global Engineering Education } \\
\text { Conference }\end{array}$ & 2 \\
\hline $\begin{array}{l}\text { IEEE Journal of Selected Topics in Signal } \\
\text { Processing }\end{array}$ & 2 \\
\hline $\begin{array}{l}\text { International Conference on Multimedia } \\
\text { Big Data (BigMM) }\end{array}$ & 2 \\
\hline
\end{tabular}

Na Tabela 2 é possível constatar que a International Conference on Learning Analytics And Knowledge (LAK) é o evento que possui o maior número de pesquisas que relatam o uso de learning analytics em MOOCs.

A segunda questão investigada teve como foco identificar a quantidade de publicações por ano, buscando-se responder ao seguinte questionamento: Em que ano há mais publicações?. Na Figura 2 há a exposição do total de publicações por ano e a quantidade por bases de dados.

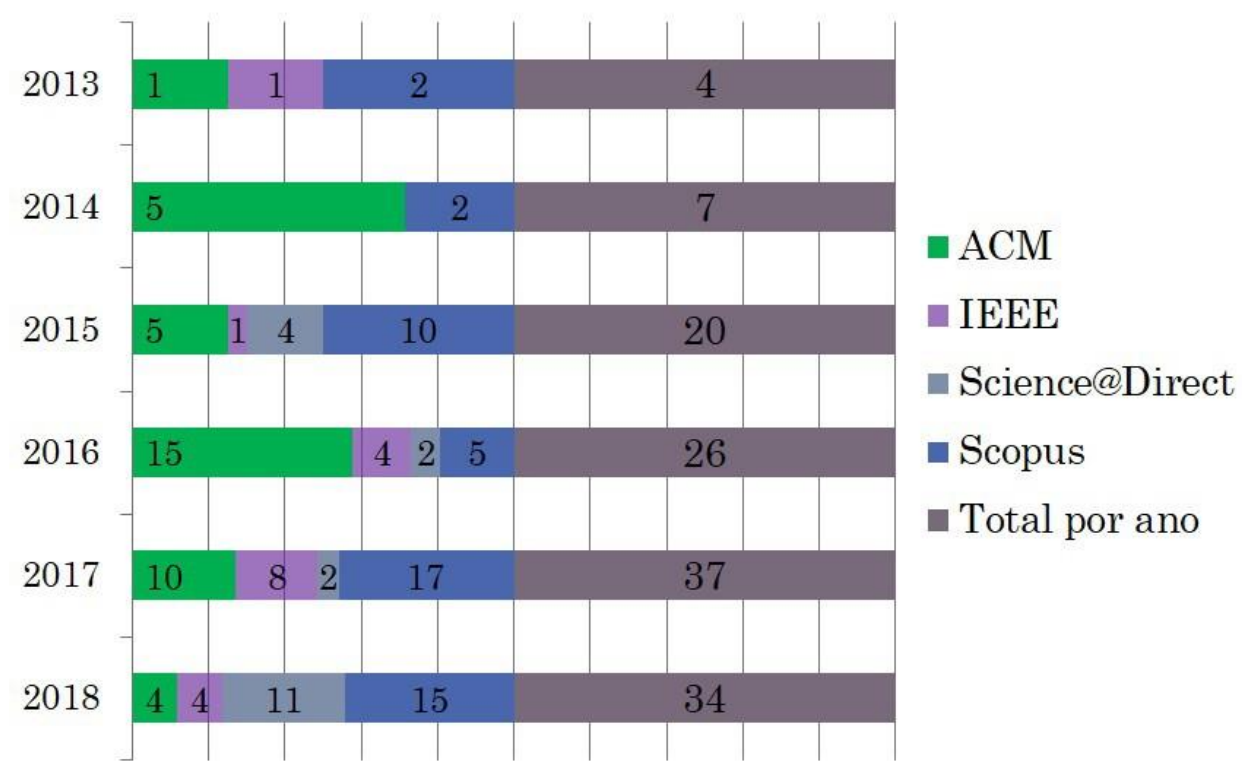

Figura 2. Quantidade de publicações por ano.

Considerando que o mapeamento sistemático compreendeu o período 2012-2018, observou-se que as ocorrências de publicações que atenderam aos critérios de seleção são estudos divulgados a partir de 2013. Com base no histórico das produções científicas, obteve-se 4 pesquisas de 2013; 7 são de 2014; 20 são estudos referentes ao ano de 2015; 26 pesquisas correspondem ao ano de 2016; 37 são de 2017 e 34 pesquisas são publicações de 2018. Desta maneira, em 2017 há um número maior de publicações. 
Ao observar de forma isolada (por base de dados), pode-se identificar na Figura 2 que na ACM Digital Library há um crescimento no número de publicações em 2016. A IEEE (com 8 pesquisas) e Scopus (17 pesquisas) apresentaram mais publicações em 2017 quando comparado aos anos anteriores, e a base de dados Science@Direct aponta maior número de estudos (11 pesquisas) em 2018. Devido à busca por trabalhos ter sido finalizada em setembro de 2018, é possível que muitos estudos referentes ao ano de 2018 ainda não estavam disponibilizados nas bases de dados utilizadas. Nesse contexto, o resultado tem como referência o histórico de publicações anteriores, revelando maior número de pesquisas em 2017, sendo 37 estudos dentre os 128 trabalhos selecionados.

No que se refere à terceira questão de pesquisa: quais os propósitos gerais de uso de learning analytics em MOOCs?, foi realizada a leitura dos resumos dos artigos e a partir dos objetivos dos autores foi gerada uma lista contendo 17 propósitos gerais:

(1) Estudar o engajamento de estudantes.

(2) Análise da conclusão de cursos.

(3) Análise do comportamento de interação e de aprendizagem.

(4) Investigar o abandono de cursos.

(5) Análise de desempenho dos estudantes

(6) Examinar fóruns de discussão.

(7) Análise de vídeos de cursos.

(8) Diagnosticar a autorregulação da aprendizagem.

(9) Realizar personalização da aprendizagem.

(10) Identificar emoções em comportamentos de interação e de aprendizagem.

(11) Análise da colaboração em cursos on-line.

(12) Análise da busca por ajuda (explícita e implícita).

(13) Análise da eficácia dos estudantes.

(14) Análise da coleta de respostas corretas por estudantes.

(15) Identificação e análise de features.

(16) Medir a qualidade da aprendizagem.

(17) Identificar padrões de persistência.

A lista de propósitos obtida dos resumos dos artigos selecionados foi utilizada para extrair termos-chave para atuar como categoria, posteriormente foi contabilizado o número de trabalhos por categoria, fornecendo a resposta para a quarta questão de pesquisa: quais temáticas são investigadas com mais frequência?. Na Tabela 3 são divulgados os totais. 
Tabela 3. Totais de trabalhos por categoria temática.

\begin{tabular}{l|c}
\hline Categoria temática & Número de papers \\
\hline Engajamento & 23 \\
\hline Conclusão & 19 \\
\hline Abandono & 18 \\
\hline Comportamentos de interação e aprendizagem & 18 \\
\hline Desempenho & 15 \\
\hline Análise de Fóruns & 9 \\
\hline Análise de vídeos & 7 \\
\hline Autorregulação & 4 \\
\hline Personalização & 4 \\
\hline Emoções & 3 \\
\hline Colaboração & 2 \\
\hline Análise da busca por ajuda & 1 \\
\hline Análise da eficácia dos estudantes & 1 \\
\hline Análise de coleta de respostas corretas & 1 \\
\hline Identificação e análise de features & 1 \\
\hline Medir a qualidade da aprendizagem & 1 \\
\hline Padrões de persistência & 1 \\
\hline
\end{tabular}

Os dados da Tabela 3 indicam quea análise do engajamento dos estudantes; investigações com ênfase na conclusão e abandono de MOOCs; estudos dos comportamentos de interação e de aprendizagem; bem como a análise do desempenho de estudantes são as temáticas mais frequentes em pesquisas que utilizam learning analytics em MOOCs.

Vale ressaltar que algumas categorias temáticas possuem subcategorias, por exemplo, na categoria engajamento havia estudos sobre os níveis de engajamento do estudante, predição de engajamento, e a maioria das pesquisas realizaram descobertas de padrões de engajamento.

$\mathrm{Na}$ categoria conclusão de cursos, algumas pesquisas investigaram aspectos de interação que estão correlacionados com a conclusão, análise de variáveis indicativas de conclusão ou fatores que levam a conclusão e/ou obtenção de certificado, sendo detectado um número maior de pesquisas voltadas para a predição de conclusão de curso.

Outro tópico frequente diz respeito ao estudo de abandono do curso, nessa categoria as pesquisas investigam fatores e indicadores de abandono, identificam estudantes em risco de abandono, verificando-se maiores ocorrências de pesquisas que realizam a predição de abandono de MOOCs.

No âmbito dos comportamentos de interação e de aprendizagem foram identificadas pesquisas com ênfase na análise dos caminhos de navegação e sequências de aprendizado, havendo um maior número de pesquisas que investigam os padrões comportamentais dos estudantes.

No que se refere à análise de desempenho dos estudantes, alguns autores analisam 
como o comportamento dos estudantes impactam no desempenho, e a maioria das pesquisas executam a predição de desempenho.

As demais abordagens identificadas voltaram-se para a análise de fóruns de discussão, que incluiu a investigação do comportamento de uso e participação em fóruns de discussão de MOOCs, e identificação de padrões de participação em fóruns. Também verificou-se a existência de alguns estudos que tiveram como foco analisar o comportamento de visualização de vídeos de MOOCs.

Além das categorias temáticas mais frequentes, citadas anteriormente, verificou-se a existência de temáticas que são mais recentes, tais como a análise da autorregulação da aprendizagem, uso de learning analytics na identificação de estilos de aprendizagem e realização da personalização da aprendizagem. Havendo também pesquisas que empregam LA na detecção de emoções, e análise de variações de estados afetivos durante experiências de aprendizagem em ambientes MOOCs. Na Figura 3 há o registro do número de categorias por ano de publicação.

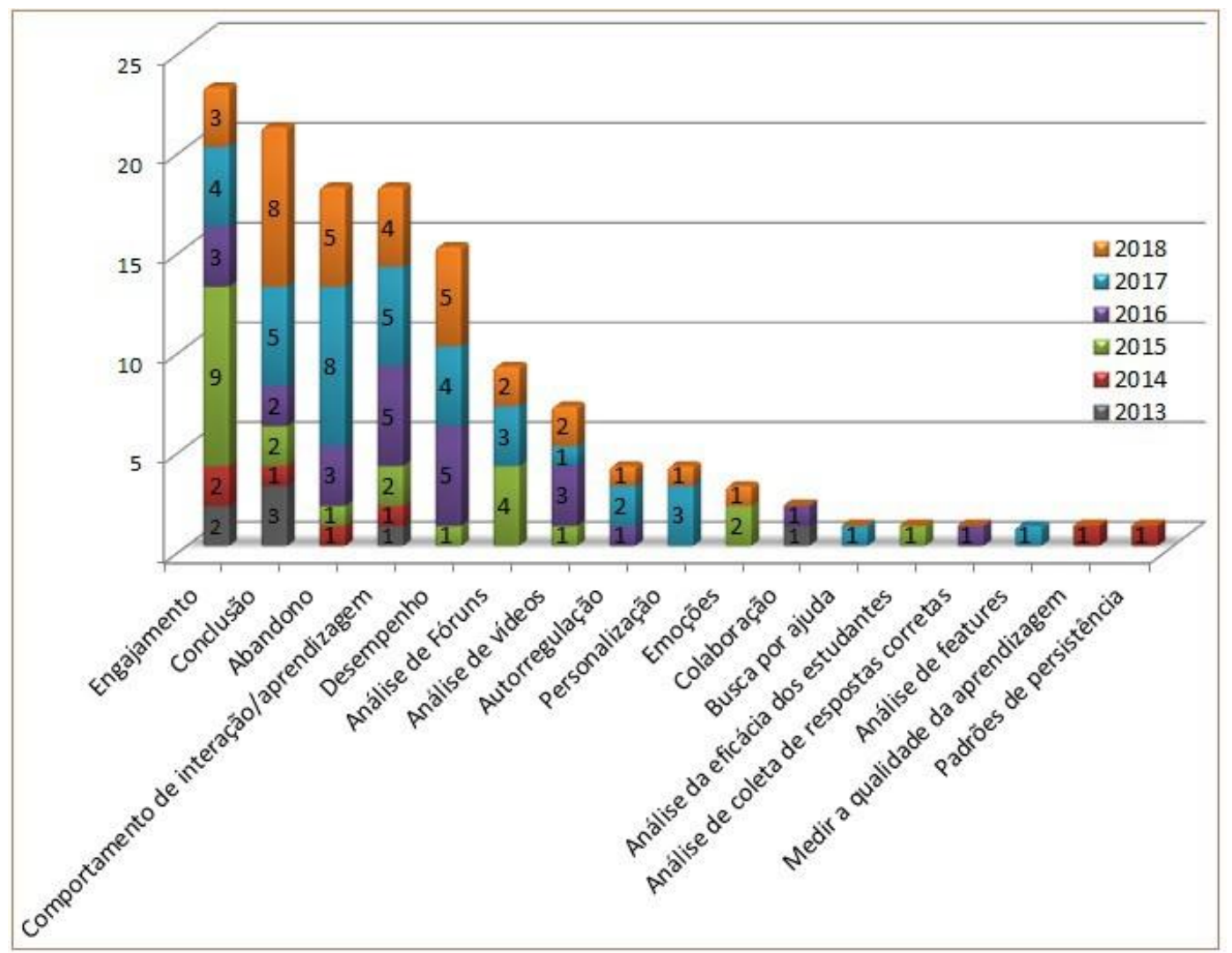

Figura 3. Tópicos temáticos por ano.

Percebe-se a partir da Figura 3 que o estudo do engajamento de estudantes de MOOCs foi iniciado em 2013, evidenciando um maior número de investigações em 2015. A análise da conclusão de MOOCs também possui registros em 2013, verificando-se o aumento no número de pesquisas sobre essa temática no decorrer dos anos, havendo mais estudos referentes ao ano de 2018.

Também foi possível identificar que o uso learning analytics para analisar o abandono de curso, investigações dos comportamentos de interação e aprendizagem de 
estudantes, bem como estudos sobre o desempenho de estudantes possuem números de registros próximos entre 2017 e 2018, revelando que esses tópicos continuam em alta.

\section{Considerações Finais}

Os MOOCs tem possibilitado a aprendizagem a uma diversidade de participantes e, simultaneamente, tem incentivado novas estratégias para melhorias do ensino $e$ aprendizagem, e a disseminação dos MOOCs tem encorajado as pessoas a se inscreverem, resultando em novas oportunidades para explorar o volume de dados registrados em plataformas de aprendizagem on-line.

Nesse contexto, nessa pesquisa foi realizado um mapeamento sistemático da literatura que objetivou verificar quais as tendências de pesquisas que empregam learning analytics em MOOCs. Os resultados mostraram que os dados de MOOCs têm sido usados para diferentes finalidades, e as técnicas de LA revelaram-se fundamentais nas tarefas de realizar descobertas e intervenções educacionais.

Os principais resultados apontaram que a análise de engajamento, conclusão e abandono de curso, investigações dos comportamentos de interação e de aprendizagem, e a análise de desempenho são as principais tendências de pesquisas, indicando ocorrências frequentes no uso de dados dos MOOCs para fins de descoberta de padrões e realização de predições.

Além dos temas predominantes, constatou-se que novas abordagens e propósitos de uso de learning analytics em MOOCs estão emergindo, destacando a importância dos dados educacionais on-line para o avanço do ensino e aprendizagem, e evidenciando as potencialidades das tecnologias na busca por conhecer os diferentes perfis e estilos de aprendizagem dos estudantes, e identificar como os conteúdos/recursos educacionais e as ações comportamentais impactam na aprendizagem.

\section{Agradecimentos}

O presente trabalho foi realizado com apoio da Coordenação de Aperfeiçoamento de Pessoal de Nível Superior - Brasil (CAPES) - Código de Financiamento 001.

\section{Referências}

CLOW, D. An overview of learning analytics. Teaching in Higher Education, v. 18, n. 6, p. 683-695, 2013.

COHEN, A.; HOLSTEIN, S. Analysing successful massive open online courses using the community of inquiry model as perceived by students. Journal of Computer Assisted Learning, p. 1-13, 2018.

CROSSLEY, S.; PAQUETTE, L.; DASCALU, M.; MCNAMARA, D. S.; BAKER, R. S. Combining Click-stream Data with NLP Tools to Better Understand MOOC Completion. Proceedings of the Sixth International Conference on Learning Analytics \& Knowledge, Edinburgh, United Kingdom, p. 6-14, 2016.

DRACHSLER, H.; KALZ, M. The MOOC and Learning Analytics Innovation Cycle MOLAC: A Reflective Summary of Ongoing Research and Its Challenges. Journal of Computer Assisted Learning., John Wiley \& Sons, Inc., New York, NY, USA, v. 32, n. 3, p. 281-290, jun. 2016. 
GIL-JAURENA, I.; DOMÍNGUEZ, D. Teachers roles in light of massive open online courses (MOOCs): Evolution and challenges in higher distance education. International Review of Education, v. 64, n. 2, p. 197-219, 2018.

HE, J.; BAILEY, J.; RUBINSTEIN, B. I. P.; ZHANG, R. Identifying At-Risk Students in Massive Open Online Courses. Proceedings of the Twenty-Ninth AAAl Conference on Artificial Intelligence, p. 1749-1755, 2015.

JOO, Y. J.; SO, H.; KIM, N. H. Examination of relationships among students' selfdetermination, technology acceptance, satisfaction, and continuance intention to use KMOOCs. Computers \& Education, v. 122, p. 260-272, 2018.

KITCHENHAM, B.; CHARTERS, S. Guidelines for performing Systematic Literature Reviews in Software Engineering, 2007.

LATCHEM, C. Open Education Resources and Massive Open Online Courses. In: OPEN and Distance Non-formal Education in Developing Countries. Singapore: Springer Singapore, 2018. p. 37-45.

LEE, Y. Using Self-Organizing Map and Clustering to Investigate Problem Solving Patterns in the Massive Open Online Course: An Exploratory Study. Journal of Educational Computing, p. 1-20, 2018.

LU, X.; WANG, S.; HUANG, J.; CHEN, W.; YAN, Z. What Decides the Dropout in MOOCs? In: DATABASE Systems for Advanced Applications. Cham: Springer International Publishing, 2017. p. 316-327.

PETERSEN, K.; FELDT, R.; MUJTABA, S.; MATTSSON, M. Systematic Mapping Studies in Software Engineering. Proceedings of the 12th International Conference on Evaluation and Assessment in Software Engineering, BCS Learning \& Development Ltd., Italy, p. 68-77, 2008.

PURSEL, B.; ZHANG, L.; JABLOKOW, K.; CHOI, G.; VELEGOL, D. Understanding MOOC students: motivations and behaviours indicative of MOOC completion. Journal of Computer Assisted Learning, v. 32, n. 3, p. 202-217, 2016.

RUIPÉREZ-VALIENTE, J. A.; MUÑOZ-MERINO, P. J.; LEONY, D.; KLOOS, C. D. ALAS-KA: A learning analytics extension for better understanding the learning process in the Khan Academy platform. Computers in Human Behavior, v. 47, p. 139-148, 2015.

SIEMENS, G. Learning Analytics: The Emergence of a Discipline. American Behavioral Scientist, v. 57, n. 10, p. 1380-1400, 2013.

ZIELINSKI, F. D. C.; SCHMITT, M. A. R. Uma ferramenta gráfica para suporte à atividade docente no Moodle. RENOTE, v. 13, n. 1, p. 1-10, 2015. 